\title{
BMJ Open Mobile phone text messages for improving adherence to antiretroviral therapy (ART): an individual patient data meta-analysis of randomised trials
}

\author{
Lawrence Mbuagbaw, ${ }^{1,2,3}$ Mia L van der Kop, ${ }^{4,5}$ Richard T Lester, ${ }^{4,6,7}$ \\ Harsha Thirumurthy, ${ }^{8}$ Cristian Pop-Eleches, ${ }^{9}$ Chenglin Ye, ${ }^{1,2}$ Marek Smieja, ${ }^{1,10}$ \\ Lisa Dolovich, ${ }^{1,10,11,12}$ Edward J Mills, ${ }^{13}$ Lehana Thabane ${ }^{1,2,12,14,15}$
}

To cite: Mbuagbaw L, van der Kop ML, Lester RT, et al. Mobile phone text messages for improving adherence to antiretroviral therapy (ART): an individual patient data meta-analysis of randomised trials. BMJ Open 2013;3: e003950. doi:10.1136/ bmjopen-2013-003950

- Prepublication history and additional material for this paper is available online. To view these files please visit the journal online (http://dx.doi.org/10.1136/ bmjopen-2013-003950).

Received 4 September 2013 Revised 4 November 2013 Accepted 6 November 2013

CrossMark

For numbered affiliations see end of article.

Correspondence to Dr Lawrence Mbuagbaw; mbuagblc@mcmaster.ca

\section{ABSTRACT}

Objectives: Our objectives were to analyse the effects of text messaging versus usual care in improving adherence to antiretroviral therapy (ART) in people living with HIV using individual patient data meta-analysis. Adjusted, sensitivity and subgroup analyses were conducted.

Setting: 3 randomised controlled trials conducted between 2010 and 2012 in rural and urban centres in Cameroon and Kenya (two studies) were used.

Participants: A total of 1166 participants were included in this analysis (Cameroon=200; Kenya=428 and 538).

Primary and secondary outcomes: The primary outcome was adherence to ART > $95 \%$. The secondary outcomes were mortality, losses to follow-up, transfers and withdrawals.

Results: Text messaging improved adherence to ART (OR 1.38; $95 \%$ Cls 1.08 to 1.78; $p=0.012$ ), even after adjustment for baseline covariates (OR 1.46; 95\% $\mathrm{Cl} 1.13$ to $1.88 ; \mathrm{p}=0.004)$. Primary education (compared with no formal education) was associated with a greater intervention effect on adherence (OR 1.65; 95\% Cl 1.10 to 2.48; $\mathrm{p}=0.016$ ) and also showed a significant subgroup effect ( $p=0.039$ ). In sensitivity analysis, our findings were robust to a modified threshold of adherence, multiple imputation for missing data and aggregate level data pooling, but not to fixed-effects meta-analyses using generalised estimation equations. There was a significant subgroup effect for long weekly ( $p=0.037$ ), short weekly text messages $(p=0.014)$ and interactive messaging $(p=0.010)$. Text messaging did not significantly affect any of the secondary outcomes.

Conclusions: Text messaging has a significant effect on adherence to ART, and this effect is influenced by level of education, gender, timing (weekly vs daily) and interactivity. We recommend the use of interactive weekly text messaging to improve adherence to ART, which is most effective in those with at least a primary level of education.

\section{INTRODUCTION}

Adherence to prescribed medication is a key principle of medical care. ${ }^{1}$ This is especially

\section{Strengths and limitations of this study}

- We employed robust analytical strategies that incorporate the within-study and between-study differences.

- The interventions use text-messaging communication in different ways (content, frequency and interactivity).

- The studies included were conducted in subSaharan Africa where HIV infection is most prevalent.

true with HIV infection, where poor adherence to antiretroviral therapy (ART) can lead to drug resistance, AIDS and subsequent morbidity and mortality. ${ }^{2}{ }^{3}$ Other consequences of suboptimal adherence include increased transmissibility of the virus and greater health system costs associated with more frequent and longer hospital stays. ${ }^{3} 4$ With more that 30 million people living with HIV worldwide, of which close to half are already on $\mathrm{ART}^{5}$ there is a need to ensure that ART is taken consistently.

The widespread use of mobile phones, including in resource-limited settings, has led to numerous studies investigating how they can be used to improve healthcare delivery. Many mobile health interventions have been based on text messaging, an inexpensive way to enhance communication between patients and healthcare personnel. ${ }^{6} \quad 7$ Evidence from two Kenyan trials suggests that mobile phone text messaging can improve adherence to ART, reduce viral load and reduce treatment interruptions ${ }^{8}{ }^{9}$; however, a third trial in Cameroon did not show significant improvement on adherence. ${ }^{10}$ These trials suggest that a number of factors, such as age, gender, level of education, duration on treatment and specific characteristics of the text messages such as interactivity, timing and 
content can influence an intervention's efficacy. Differences in effectiveness between and within studies can best be investigated using individual patient data (IPD) meta-analysis. This meta-analysis synthesises the three trials using mobile phone interventions for ART adherence and examines their effectiveness in important subgroups. $^{11}$

\section{Objectives}

The objectives of this IPD meta-analysis are to

1. Summarise the evidence from three trials on the use of mobile phone text messaging compared with usual care to improve adherence to ART in people;

2. Analyse the effect of the intervention in subgroups defined by age, gender, level of education, duration on ART and type of message;

3. Examine the use of multiple statistical methods and their effects on outcomes.

\section{METHODS}

The methods have been documented in a published protocol. $^{12}$ In brief, we conducted an IPD meta-analysis of three randomised controlled trials (RCTs) investigating the use of text messaging to improve adherence to ART. The first of these was the WelTel Kenyal RCT that was a multisite two-arm trial of weekly interactive text messaging versus usual care conducted in Kenya. ${ }^{8}$ The second trial was a single site five-arm trial conducted in Kenya that compared short daily, short weekly, long daily and long weekly one-way text messages to usual care in a five-arm trial in Kenya. ${ }^{9}$ The two Kenyan trials ran for 12 months. The third trial was the Cameroon Mobile Phone SMS (CAMPS) trial, which was a single site two-arm trial of weekly motivational text messaging versus usual care to improve adherence to ART over 6 months. ${ }^{10}$ The first two trials showed significant improvement in adherence to ART while the latter did not. These were the only trials identified by the Adherence Trialists Collaboration and a PubMed search for RCTs of text messaging for ART adherence.

\section{Data management}

Only anonymised data were collected and used for this study. Data from all three trials were recoded and merged in a safe and secure computer system with backup. After merging, data were compared with the published manuscripts to ensure accuracy. Only variables available in all three data sets were used. Similar variables were pooled together. Categorical variables were modified to create new uniform categories across all studies. For example, categories such as 'lower primary', 'upper primary' and 'completed primary' were merged into 'primary education'. A new data set was created with the following baseline covariates: age (years), gender (male/female), level of education (none, primary, secondary or higher) and duration on ART (months). These covariates were used for the subgroup analysis. The intervention was defined as text messaging versus no text messaging. For the subgroup analysis, text messaging was further divided into short daily, ${ }^{9}$ long daily, ${ }^{9}$ short weekly ${ }^{89}$ and long weekly messages ${ }^{910}$, motivational $^{9}{ }^{10}$ versus non-motivational ${ }^{8}$ and interactive, ${ }^{8} \quad 10$ versus non-interactive. ${ }^{9}$ Motivational messages were considered as those with words of encouragement. The primary outcome was adherence greater than $95 \%$ as measured by the authors. In other words, irrespective of how adherence was measured participants whose level of adherence was rated above $95 \%$ were considered to have achieved the primary outcome. Two of these trials used self-reported adherence, ${ }^{810}$ while the third used medication event monitoring system (MEMS) caps. ${ }^{9}$ The secondary outcomes were mortality, and the number of participants lost to follow-up, transferred out or withdrawn.

\section{Statistical methods}

Baseline data for all included participants were summarised as mean (SD) or median (first and third quartiles, Q1, Q3) for continuous variables and number (\%) for categorical variables. The primary outcome was adherence to ART (defined as adherence above 95\%). We used IPD random-effects meta-analysis to determine the effects of text messaging on the primary outcome. In this model, each study was considered as a random effect. ORs and 95\% CIs are reported. The level of statistical significance was set at $\alpha=0.05$. This primary analytical method was repeated for the secondary outcomes.

In an adjusted analysis, we investigated the effects of baseline covariates (age, gender, level of education and duration on ART) on the primary outcome. They were inserted in the model as fixed effects.

We also conducted four sensitivity analyses: (1) we changed the method of analysis (using generalised estimation equations (GEE)) for exploratory purposes; (2) we changed the threshold for adherence $(>90 \%)$, as the recent literature suggests that viral load suppression can be achieved at lower levels of adherence ${ }^{13}{ }^{14}$; (3) we used multiple imputation techniques to deal with missing data; and (4) we calculated pooled estimates of effect (aggregate data meta-analysis). We present heterogeneity statistics and forest plots for the aggregate data meta-analysis.

In addition, we looked at the effects of text messaging in different subgroups defined by age, gender, level of education, duration on ART, type of message (short daily, long daily, short weekly and long weekly), content of message (motivational vs non-motivational) and interactivity (two-way vs one-way). The $\mathrm{p}$ values of the interaction terms are reported. Data were analysed using Statistical Analysis Software (SAS) V.9.2 (SAS Institute, Cary, North Carolina, USA, 2009).

\section{RESULTS}

\section{Baseline characteristics}

The three trials included 1166 participants. Approximately two-thirds $(67.0 \%)$ were female, and almost half $(47.5 \%)$ had above a primary level of education. The median age 
Table 1 Baseline characteristics of participants in the three text-messaging adherence trials

\begin{tabular}{|c|c|c|c|}
\hline & $\begin{array}{l}\text { Control arm }(n=663) \\
n(\%)\end{array}$ & $\begin{array}{l}\text { SMS arm }(n=503) \\
n(\%)\end{array}$ & $\begin{array}{l}\text { Total }(n=1166) \\
n(\%)\end{array}$ \\
\hline \multicolumn{4}{|l|}{ Categorical variables } \\
\hline \multicolumn{4}{|l|}{ Number of participants } \\
\hline Lester et $a \beta^{\beta}$ & 265 (22.7) & $273(23.4)$ & $538(46.1)$ \\
\hline Pop-Eleches et å & 139 (11.9) & $289(24.8)$ & $428(36.7)$ \\
\hline Mbuagbaw et $a l^{10}$ & 99 (8.5) & $101(8.7)$ & $200(17.2)$ \\
\hline \multicolumn{4}{|l|}{ Gender* } \\
\hline Male & $159(32.7)$ & 207 (33.2) & 366 (33.0) \\
\hline Female & 327 (67.3) & $416(66.8)$ & $743(67.0)$ \\
\hline \multicolumn{4}{|l|}{ Level of education* } \\
\hline None & $70(14.4)$ & $121(19.4)$ & $191(17.2)$ \\
\hline Primary & $164(33.7)$ & $227(36.4)$ & 391 (35.3) \\
\hline Secondary or higher & $252(51.9)$ & $275(44.1)$ & $572(47.5 \%)$ \\
\hline Continuous variables & Mean (SD) & $\operatorname{Mean}(S D)$ & Mean (SD) \\
\hline Duration on ART (months) $†$ & $5.99(16.00)$ & $5.73(15.342)$ & $5.84(15.626)$ \\
\hline Age (years) $\ddagger$ & 37.19 (9.192) & $37.33(9.476)$ & $37.27(9.348)$ \\
\hline
\end{tabular}

was 36 years (range=66), and the mean duration on ART was 5.8 months $(\mathrm{SD}=15.63)$. The details of the baseline characteristics are reported in table 1 .

\section{Primary analysis}

In this random effects meta-analysis SMS text messaging significantly improved adherence to ART above $95 \%$ (OR $1.38,95 \%$ CI 1.08 to $1.78, \mathrm{p}=0.012$; see figure 1 ).

\section{Adjusted analysis}

In the adjusted analysis, female versus male gender (OR $1.38,95 \%$ CI 1.05 to $1.82 ; \mathrm{p}=0.022)$ and primary education compared with no formal education (OR 1.65, 95\% CI 1.10 to $2.48 ; \mathrm{p}=0.016$ ) were significant predictors of adherence $>95 \%$. Age and duration on ART were not significantly associated with better adherence (see figure 1).

\section{Sensitivity analysis}

Text messaging did not significantly improve adherence to ART in the sensitivity analysis using GEE (OR 1.11, $95 \%$ CI 0.88 to $1.41 ; \mathrm{p}=0.373)$. The intraclass correlation coefficient for the primary outcome was 0.18 , suggesting considerable differences between the studies. When we considered a threshold of $90 \%$ for adherence (OR 1.51, 95\% CI 1.07 to 2.13 ; $\mathrm{p}=0.018$ ), used multiple imputation techniques (we created five data sets of plausible values that were merged into one for analysis) to replace missing data (OR 1.34, 95\% CI 1.05 to $1.72 ; \mathrm{p}=0.021$ ) or conducted a random-effects aggregate data meta-analysis (OR $1.35,95 \%$ CI 1.05 to $1.73, \mathrm{p}=0.020, \tau^{2}=0 ; \mathrm{I}^{2}=0 \%$ ) text messaging had a positive effect on adherence to ART. These results are displayed in figure 1.

\section{Subgroup analyses}

In the subgroup analyses primary education $(\mathrm{p}=0.039)$, long weekly $(\mathrm{p}=0.037)$, short weekly text messages $(\mathrm{p}=0.014)$ and interactive messages $(\mathrm{p}=0.010)$ had significant positive interactions with text messaging. Age, gender, duration on ART and motivational content showed no subgroup effects. These results are summarised in figure 1 .

\section{Secondary outcomes}

Text messaging did not significantly reduce mortality or losses to follow-up. Transfers and withdrawals were also not significantly affected by text messaging (see table 2).

\section{DISCUSSION}

This IPD meta-analysis of three trials found that text messaging had a positive effect on adherence to ART. This effect is stronger in women and in those with a primary education. Weekly and interactive messages are also more effective. Our findings confirm the efficacy of text messaging in improving adherence to ART but demonstrate that this effect may be nuanced and work for some patients more so than others. While there is widespread support for text messaging, our analysis suggests guidelines should be specific about what design text messaging interventions should be implemented. ${ }^{15} 16$

Three issues stand out in this analysis. First, primary education stood out in the adjusted and subgroup analysis as an important factor influencing the effect of text messaging on ART. Understandably, education is related to literacy and consequently the ability to read text messages. It would seem, however, that beyond primary education, there are no further benefits to be gained, as can be seen in the subgroup analysis, where there was no 
Figure 1 Forest plot for primary, adjusted, sensitivity and subgroup analysis of text messaging to improve adherence to ART. ART levels of education are compared with no formal education; types of text messages are compared with control; $3.3 \%$ missing data for primary outcome. ART, antiretroviral therapy; GEE, generalised estimation equations; MI, multiple imputation; RE, random-effects.

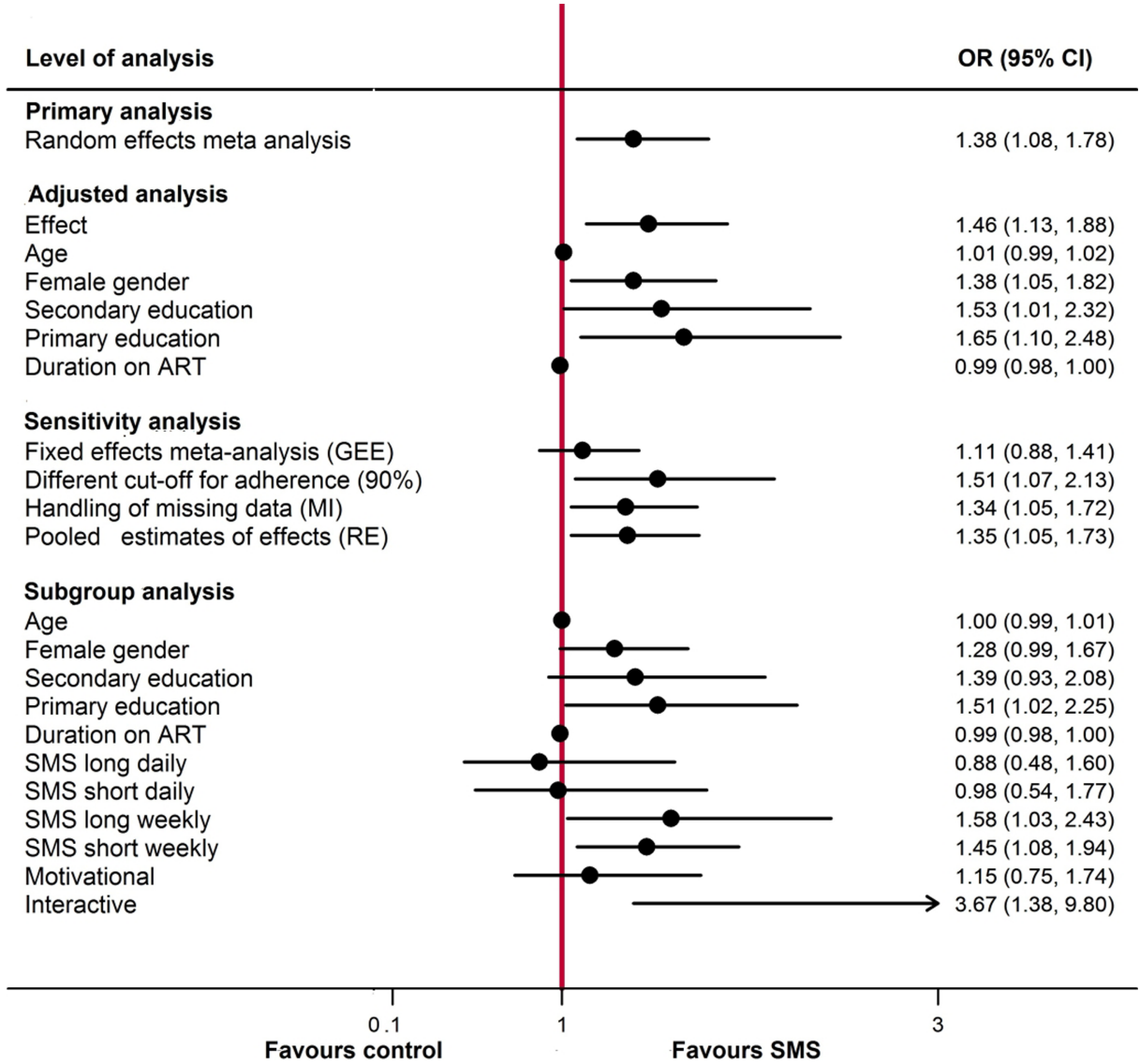

Favours control interaction with secondary education. The potential marginalisation of people with limited literacy with regard to text messaging has been described in other studies. $^{11} 17 \quad 18$ Second, the subgroups that received weekly messages were more likely to achieve adherence above $95 \%$. This may be because daily messages are intrusive, cause user fatigue and therefore rendered ineffective. However, these analyses should be treated with caution, as the data for daily messages came from only one of the trials. ${ }^{9}$ Further research is necessary to understand the role of timing on the efficacy of text messaging.

Third, text messaging did not have a significant effect when we used GEE. A GEE approach estimates population averaged effects, and the estimate of SE is very variable when there are few clusters. ${ }^{19}$ In this IPD analysis with only three clusters, GEE may not be the appropriate parameter estimator.

Table 2 Summary of results for secondary outcomes

\begin{tabular}{lll}
\hline Secondary outcomes & OR $(95 \%$ Cl $)$ & p Value \\
\hline Mortality & $0.87(0.52$ to 1.43$)$ & 0.591 \\
Lost to follow-up & $0.87(0.63$ to 1.19$)$ & 0.387 \\
Transfers & $1.40(0.56$ to 1.48$)$ & 0.463 \\
Withdrawals & $2.55(0.67$ to 9.71$)$ & 0.170 \\
\hline
\end{tabular}

In addition, motivational content seemed to provide no additional value, and may not be necessary in many cases. On the other hand, interactivity boosted the intervention effect and should be encouraged. Still, we advise caution with this interpretation as the effect of interactivity may be a between-study difference. Data for interactivity came from all the participants in the two trials. $^{8} 10$

This study is not without limitations, notably the fact that the three studies included in this review were conducted in Africa and therefore these findings may not be applicable to other settings. However, this is also strength as adherence enhancing interventions are particularly relevant to Africa, the heart of the HIV pandemic. Combining widely variable text messaging interventions in this meta-analysis may also limit the interpretation of our findings. The generalisability of our findings may also be affected by the differences in the way adherence was measured in the included studies and the average duration on ART (about 6 months); however, our primary choice of analytical method was meant to account for these differences between studies. Even though we did not conduct a systematic search for other RCTs of text messaging to improve adherence to ART, we reached out to the Adherence Trialists Collaboration to identify other trials and none were identified. Other such RCTs, if any, can be included in subsequent iterations of these analyses. Only one of the studies used viral load as a measure of adherence. It is 
possible our results would be different had we had access to viral load data.

The strengths of this analysis include the use of IPD, an analysis of whether text messages are more effective in patients with certain characteristics, the use of robust statistical techniques to account for within-study and between-study differences, and our focus on Africa where our research is most needed.

\section{CONCLUSION}

Test messaging has a significant effect on adherence to ART. We recommend the use of interactive weekly text messaging to boost adherence to ART, especially in clients with at least a primary level of education. Further research endeavours should investigate the cost-effectiveness of textmessaging interventions and approaches to scaling up.

\section{Author affiliations}

${ }^{1}$ Department of Clinical Epidemiology and Biostatistics, McMaster University, Hamilton, Ontario, Canada

'Biostatistics Unit, Father Sean O'Sullivan Research Centre, St Joseph's Healthcare, Hamilton, Ontario, Canada

${ }^{3}$ Centre for Development of Best Practices in Health, Yaounde Central Hospital, Yaounde, Cameroon

${ }^{4}$ Department of Medicine, University of British Columbia, Vancouver, British Columbia, Canada

${ }^{5}$ Department of Public Health Sciences, Karolinska Institutet, Stockholm, Sweden

${ }^{6}$ British Columbia Centre for Disease Control, British Columbia, Canada ${ }^{7}$ Department of Medical Microbiology, University of Manitoba/University of Nairobi, Nairobi, Kenya

${ }^{8}$ Department of Health Policy and Management, Gillings School of Global Public Health, University of North Carolina at Chapel Hill, Chapel Hill, North Carolina, USA

${ }^{9}$ School of International and Public Affairs, Columbia University, New York, New York, USA

${ }^{10}$ St. Joseph's Healthcare Hamilton, Ontario, Canada

${ }^{11}$ Department of Family Medicine, McMaster University, McMaster Innovation Park, Hamilton, Ontario, Canada

${ }^{12}$ Centre for Evaluation of Medicine, St Joseph's Healthcare, Hamilton, Ontario, Canada

${ }^{13}$ Faculty of Health Sciences, University of Ottawa, Ottawa, Ontario, Canada

${ }^{14}$ Departments of Paediatrics and Anaesthesia, McMaster University, Hamilton, Ontario, Canada

${ }^{15}$ Population Health Research Institute, Hamilton Health Sciences, Hamilton, Ontario, Canada

Contributors All the authors participated in planning and conducting the study, and approved the final version. LM and LT conceived of the idea. LM wrote the first draft of this manuscript. MLvdK, RTL, HT, CP-E, MS, LD, EJM and $C Y$ revised several versions of the manuscript. CY conducted the statistical analysis.

Competing interests RTL is the founder of WelTel, a non-profit organisation designed to scale-up evidence-based mHealth interventions. MLV is supported by a Canadian Institutes of Health Research Doctoral AwardDoctoral Foreign Study Award (October 2012), offered in partnership with the CIHR Strategy for Patient-Oriented Research and the CIHR HIV/AIDS Research Initiative. RTL is supported in part by an mHealth grant from Grand Challenges Canada.

Ethics approval This is a secondary analysis of data.

Provenance and peer review Not commissioned; externally peer reviewed.

Data sharing statement No additional data are available.
Open Access This is an Open Access article distributed in accordance with the Creative Commons Attribution Non Commercial (CC BY-NC 3.0) license, which permits others to distribute, remix, adapt, build upon this work non-commercially, and license their derivative works on different terms, provided the original work is properly cited and the use is non-commercial. See: http://creativecommons.org/licenses/by-nc/3.0/

\section{REFERENCES}

1. Osterberg L, Blaschke T. Adherence to medication. $N$ Engl J Med 2005;353:487-97.

2. WHO. Adherence to long-term therapies: evidence for action. Secondary adherence to long-term therapies: evidence for action 2003. http://www.who.int/chp/knowledge/publications/adherence_ report/en/

3. Paterson DL, Swindells S, Mohr J, et al. Adherence to protease inhibitor therapy and outcomes in patients with HIV infection. Ann Intern Med 2000;133:21-30.

4. Knobel $\mathrm{H}$, Codina $\mathrm{C}$, Miro JM, et al. [The recommendations of GESIDA/SEFH/PNS for improving adherence to antiretroviral treatment. AIDS Study Group of the Spanish Society of Hospital Pharmacy and the National Plan on AIDS of the Minister of Health and Consumers]. Enferm Infecc Microbiol Clin 2000;18:27-39.

5. UNAIDS. Report on the Global AIDS Epidemic. Secondary Report on the Global AIDS Epidemic 2010. http://www.unaids.org/ globalreport/global_report.htm

6. Mukund Bahadur KC, Murray PJ. Cell phone short messaging service (SMS) for HIV/AIDS in South Africa: a literature review. Stud Health Technol Inform 2010;160(Pt 1):530-4.

7. Mbuagbaw L, Thabane L, Ongolo-Zogo P. Opening communication channels with people living with HIV using mobile phone text messaging: insights from the CAMPS trial. BMC Res Notes 2013;6:131.

8. Lester RT, Ritvo P, Mills EJ, et al. Effects of a mobile phone short message service on antiretroviral treatment adherence in Kenya (WelTel Kenya1): a randomised trial. Lancet 2010;376:1838-45.

9. Pop-Eleches C, Thirumurthy H, Habyarimana JP, et al. Mobile phone technologies improve adherence to antiretroviral treatment in a resource-limited setting: a randomized controlled trial of text message reminders. AIDS 2011;25:825-34.

10. Mbuagbaw L, Thabane L, Ongolo-Zogo $\mathrm{P}$, et al. The Cameroon Mobile Phone SMS (CAMPS) trial: a randomized trial of text messaging versus usual care for adherence to antiretroviral therapy. PLOS ONE 2012; 7:e46909.

11. Cole-Lewis H, Kershaw T. Text messaging as a tool for behavior change in disease prevention and management. Epidemiol Rev 2010;32:56-69.

12. Mbuagbaw L, van der Kop ML, Lester RT, et al. Mobile phone text messages for improving adherence to antiretroviral therapy (ART): a protocol for an individual patient data meta-analysis of randomised trials. BMJ Open 2013;3:e002954.

13. Bangsberg DR. Less Than $95 \%$ adherence to nonnucleoside reverse-transcriptase inhibitor therapy can lead to viral suppression. Clin Infect Dis 2006;43:939-41.

14. Apisarnthanarak A, Mundy LM. Long-term outcomes of HIV-infected patients with $<95 \%$ rates of adherence to nonnucleoside reverse-transcriptase inhibitors. Clin Infect Dis 2010;51:115-17.

15. Horvath T, Azman H, Kennedy GE, et al. Mobile phone text messaging for promoting adherence to antiretroviral therapy in patients with HIV infection. Cochrane Database Syst Rev 2012;3 CD009756.

16. Thompson MA, Aberg JA, Hoy JF, et al. Antiretroviral treatment of adult HIV infection: 2012 recommendations of the International Antiviral Society-USA panel. JAMA 2012;308:387-402.

17. Siedner MJ, Haberer JE, Bwana MB, et al. High acceptability for cell phone text messages to improve communication of laboratory results with HIV-infected patients in rural Uganda: a cross-sectional survey study. BMC Med Inform Decis Mak 2012;12:56.

18. Person AK, Blain ML, Jiang $\mathrm{H}$, et al. Text messaging for enhancement of testing and treatment for tuberculosis, human immunodeficiency virus, and syphilis: a survey of attitudes toward cellular phones and healthcare. Telemed $J$ E Health 2011:17:189-95.

19. Ma J, Thabane L, Kaczorowski J, et al. Comparison of Bayesian and classical methods in the analysis of cluster randomized controlled trials with a binary outcome: the Community Hypertension Assessment Trial (CHAT). BMC Med Res Methodol 2009;9:37. 